
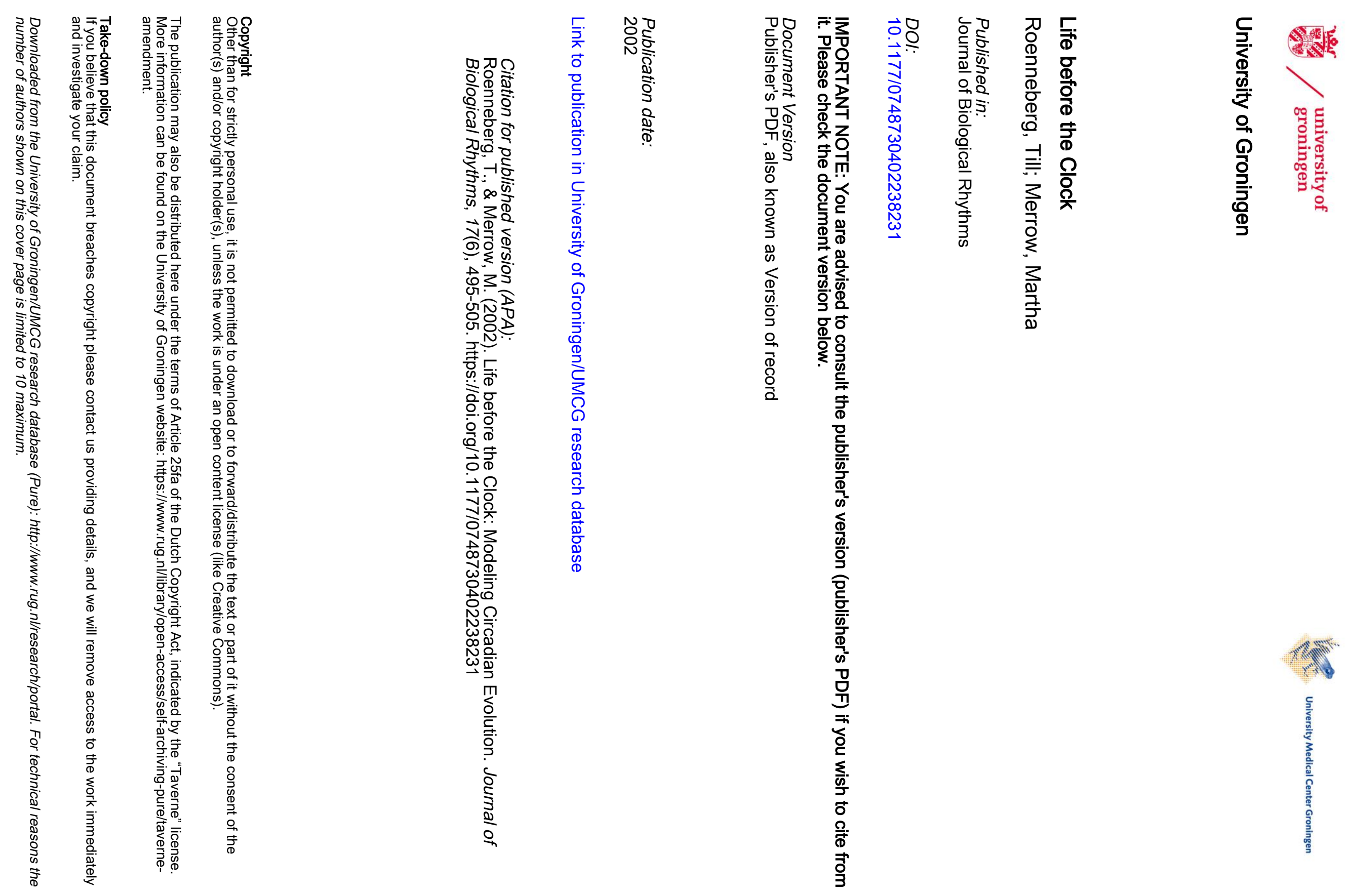


\title{
ARTICLE
}

\section{Life before the Clock: Modeling Circadian Evolution}

\author{
Till Roenneberg ${ }^{1}$ and Martha Merrow \\ Institute for Medical Psychology, Chronobiology Division, \\ Ludwig-Maximilians-Universität München, D-80336 Munich, Germany
}

\begin{abstract}
A feedback loop that functions via transcription and translation is thought to be the mechanistic core of circadian rhythmicity. Numerous modeling efforts incorporate the identified components and their modifications to recreate the circadian clock in computer simulations. Several issues remain problematic, including the lack of precise quantitative kinetics and the likely existence of additional, as-yet-undiscovered components. Even without these complications, models and flow charts of the circadian system have reached high complexity. They attempt to reconcile all observations without violating current views and concepts. In this article, the authors consider the mechanisms that may have preceded the circadian system in evolution. Given that cellular metabolism and biochemistry were presumably already interconnected in cascading feedback reactions prior to the appendage of the transcription/translation feedback loop, a coordinated response to exogenous changes would be advantageous over unsystematic responses. The authors hypothesize that those mechanisms that allowed synchronization in spite of metabolic complexity form the basis for the evolution of circadian properties and are as fundamental to the circadian system as the transcriptional/translational feedback loop.
\end{abstract}

Key words circadian rhythm, model, evolution, negative feedback

In 1729, the French astronomer DeMairan discovered that leaf movements in plants continue with a 24-h rhythmicity in constant darkness. Since then, the description of the mechanism responsible for endogenous, circadian rhythms has come a long way. Mainly from the work of Bünning, Aschoff, and Pittendrigh, we know the formalisms and rules of circadian timing. The anatomical location of the central circadian pacemaker is known for most animals (e.g., the suprachiasmatic nucleus [SCN]), whereas plants appear to lack central pacemakers. Circadian rhythmicity is a property of most cells, and a network of clock genes is being discovered.

The current conceptual view of the molecular circadian clock mechanism is based on the hypothesis that circadian rhythmicity is generated by a negative feedback loop that is closed via the expression of clock genes and their protein products (a transcriptionaltranslational loop [TTL]). This model was originally hypothesized for the Drosophila period gene (Hardin et al., 1990) and was strengthened by the fact that the Neurospora clock gene, frequency, and its protein also

1. To whom all correspondence should be addressed: Institute for Medical Psychology, Chronobiology Division, LudwigMaximilians-Universität München, Goethestr. 31, D-80336 Munich, Germany; e-mail: till.roenneberg@imp.med. uni-muenchen.de.

JOURNAL OF BIOLOGICAL RHYTHMS, Vol. 17 No. 6, December 2002 495-505

DOI: $10.1177 / 0748730402238231$

(C) 2002 Sage Publications 
form a negative feedback (Aronson, Johnson, Loros, et al., 1994). The circadian feedback loops described in cyanobacteria (Iwasaki and Kondo, 2000; Mori and Johnson, 2001), Neurospora (Loros and Dunlap, 2001; Merrow et al., 2001), Drosophila (Blau, 2001; Williams and Sehgal, 2001), mammals (Herzog and Tossini, 2001; Reppert and Weaver, 2001), and more recently in plants (Alabadi et al., 2001) use different sets of genes. However, even loops that use the same components (like in insects and mammals) are put together differently or the respective genes and their products serve different functions. There is no doubt about the important role of these clock genes in each of the circadian systems. Without them, circadian rhythmicity lacks many of its characteristics, including selfsustainability in constant conditions (Bargiello et al., 1984; Gekakis et al., 1998; Loros and Feldman, 1986), entrainability by light (Albrecht et al., 2001; LakinThomas and Brody, 2000; Merrow et al., 1999; Stanewsky et al., 1998; Zheng et al., 2001), and temperature and nutritional compensation (Loros and Feldman, 1986). The known molecular feedback loops in different species are all tightly linked to light input pathways, which has led to the assumption that the circadian clock evolved from light transduction mechanisms (Crosthwaite et al., 1997). Yet, experiments in Neurospora and Arabidopsis show that other circadian characteristics (e.g. entrainment by temperature cycles) remain in the absence of clock genes (McWatters et al., 2000; Merrow et al., 1999), so additional components of the system are still to be identified-most probably in all model systems. A recent study strengthens the case for additional components. In cockroaches, aftereffects can only be elicited when the insects are kept for several days in light:dark cycles but not if they are submitted to temperature cycles (Page et al., 2001). These results indicate that a lightsensitive part may be distinct from a temperaturesensitive part of the circadian machinery. Our knowledge about the former is much more detailed than it is about the latter, so that each additional component will increase our understanding of the circadian machinery, especially if it opens up cellular mechanisms outside of the known TTLs.

One of the most conspicuous qualities of circadian systems is self-sustained oscillation in constant conditions. This feature was instrumental in the discovery of the circadian clock, in proving the rhythm's endogenous nature, and in identifying its genetic components. But what do we learn from experiments in constant conditions in view of the fact that the evolution of the circadian system took place predominantly under the influences of a rotating earth? Theoretically, any damped oscillator mechanism would serve just as well with zeitgebers counteracting decreasing amplitudes. It is likely that self-sustained rhythmicity is a consequence of how the system is built to function optimally under entrained conditions rather than being the object of the evolutionary process (Roenneberg and Merrow, 2001). But, what does "optimal function" of the circadian system mean in the context of a 24-h day? Circadian systems are entrained rather than driven by zeitgebers. Unlike driven synchronization, entrainment results in a specific phase relationship to the zeitgeber cycle, which depends on the system's endogenous period and its phase response curve as well as on the amplitude of both the zeitgeber and the endogenous oscillator. Entrainable systems are taken to be advantageous over purely driven ones by providing the possibility of anticipation, that is, preparing physiology before the external changes occur (sunrise, drop in temperature, food availability, etc.). Entrainable systems can vary their phase relationship within a zeitgeber cycle simply by changing the endogenous period or the strength by which the phase-resetting mechanism responds to a given zeitgeber (amplitude of the phase response curve). This provides flexibility in adjusting to different external changes. Although the sun rises at the same time each day (or predictably earlier/later within the course of the year), the timing of food sources might not always be directly linked to the earth's rotation. Yet, food availability may still be predictable from its previous spatiotemporal history. A physiology that is simply driven by the external changes would, thus, be disadvantageous relative to one that is regulated by an endogenous rhythmicity with flexible and anticipatory qualities (Daan, 1981).

An inherent assumption in this line of arguments is that the circadian system was implemented as an "improvement" over purely driven systems. However, complex networks of metabolism, which must have existed in the most primitive organisms, may not be simply drivable by environmental cycles; they may have had to develop mechanisms to counteract chaotic responses of a complex intracellular network to exogenous zeitgebers. If one presumes that such a mechanism is necessary for reliable synchronization with the environment, organisms lacking this mechanism would be at a disadvantage in a periodic world. Therefore, the foundation on which the circadian clock is built might be those mechanisms that allow 
physiology to be driven in spite of complexity. We have formalized these assumptions by modeling a circular network of feedback loops.

\section{METHODS}

Modeling biological functions can have very different goals, each with different merits for understanding an initial "back-box system." Either they may attempt to mimic experimental results as closely as possible (Leloup and Goldbeter, 1998) or they can be a formal basis for hypotheses (e.g., the existence of singularity) (Winfree, 1970) that can then be verified experimentally (Taylor et al., 1982). Here, we use mathematical modeling to explore a possible scenario of circadian evolution. The model is essentially a coupled oscillator system. Several models of the circadian system have used multiple coupled oscillators, for example, to predict the interaction by global coupling between neurons within the SCN and their contribution to the in-toto phenotype (Achermann and Kunz, 1999). Other coupled oscillator models investigate the interaction between bilateral pacemakers (Petri and Stengl, 2001), between subpopulations of SCN neurons, or between the evening and morning oscillator (Daan et al., 2001; Oda et al., 2000). This article does not attempt to compare its algorithms with previous multioscillator models. Its major difference is that the underlying oscillators, in isolation, do not have periods in the circadian range and are not self-sustained. The model connects five oscillators in a circuit, which couples the individual components either by direct coupling (i.e., the product of one of the feedback reactions influences the kinetics of the next) or by a global coupling (i.e., the summed output of each feedback loop influences one of the components).

Models were implemented with the help of the Stella ${ }^{\circledR}$ Program for Macintosh, and model results were further analyzed and graphed with the help of the CHRONO program (Roenneberg and Taylor, 2000). All feedback loops ( $F B_{1}$ to $F B_{5}$; see Fig. 1A) used in the network are based on the same set of equations describing the concentration changes of two state variables $\left(S_{1}\right.$ and $\left.S_{2}\right)$ over time (see Table 1$)$. The two "reactions" form a negative feedback loop by $S_{2}$ inhibiting the production of $S_{1}$. The algorithms used for the feedback loops are essentially the same as used in the previously published single feedback models (Roenneberg and Merrow, 1998, 1999):

$$
\begin{aligned}
& S_{1}(t)=S_{1}(t-d t)+\left(p_{1}-d_{1}\right) * d t \\
& S_{2}(t)=S_{2}(t-d t)+\left(p_{2}-d_{2}\right) * d t .
\end{aligned}
$$

The model was run at time intervals $(d t)$ of $1 \mathrm{~h}$ (using the fourth-order Runge-Kutta integration algorithm). The production of $S_{1}\left(p_{1}\right.$; column 2 in Table 1$)$ is inhibited by $S_{2}$, whereas the production of $S_{2}\left(p_{2}\right.$; column 3 in Table 1) depends directly on the concentration of $S_{1}$. The degradation of each state variable depends on its current concentration, that is, the higher its concentration, the stronger its degradation $\left(d_{1}\right.$ and $d_{2}$; columns 5 and 6 in Table 1). The feedback loops differ only in their turnover rates of $S_{1}(r$, influencing both production and degradation; column 1 in Table 1).

A circular network between the five feedback loops is implemented by direct coupling. State variable $\left(S_{2}\right)$ of one loop influences the production of state variable $\left(S_{1}\right)$ in the next loop: $S_{2,1}$ influences $p_{1,2}, S_{2,2}$ influences $p_{1,3}, \ldots, S_{2,5}$ closes the circle by influencing $p_{1,1}(c$; column 4 in Table 1).

$F B_{1}$ constitutes the input component of the network and is influenced by the zeitgeber $(Z G)$, which affects the production of $S_{1,1}$ by adding a rate to $p_{1,1}$ for $12 \mathrm{~h}$ each $24 \mathrm{~h}$ (see figures for the different zeitgeber strength used). An integrated output of the network is modeled by simply adding the concentrations of all $S_{2}$ for a given time point $(\Sigma$; see the bottom trace in Fig. 1B). The input component, $F B_{1}$, is influenced not only by $Z G$ but also via feedback by the zeitnehmer $(Z N)$ (ZN represents the integrated output, $\Sigma$, multiplied by the zeitnehmer strength, $I_{Z N}$; see equation [3]). The degradation of $S_{2,1}$ is increased by adding $Z N$ from the previous time interval (i.e., with a delay of $1 \mathrm{~h}$; see column 6 in Table 1 and figures for the different zeitnehmer strength, $I_{Z N}$, used):

$$
Z N=I_{Z N} * \Sigma\left(S_{2,1} \text { to } S_{2,5}\right)
$$

\section{RESULTS AND DISCUSSION}

\section{Metabolic Networks}

Some kind of negative feedback is the basis of most oscillations, but negative feedback alone does not ensure self-sustained rhythmicity. In addition, negative feedback is one of the most common control mechanisms within pathways at all levels (e.g., product inhibition in enzymatic reactions, gene regulation, 


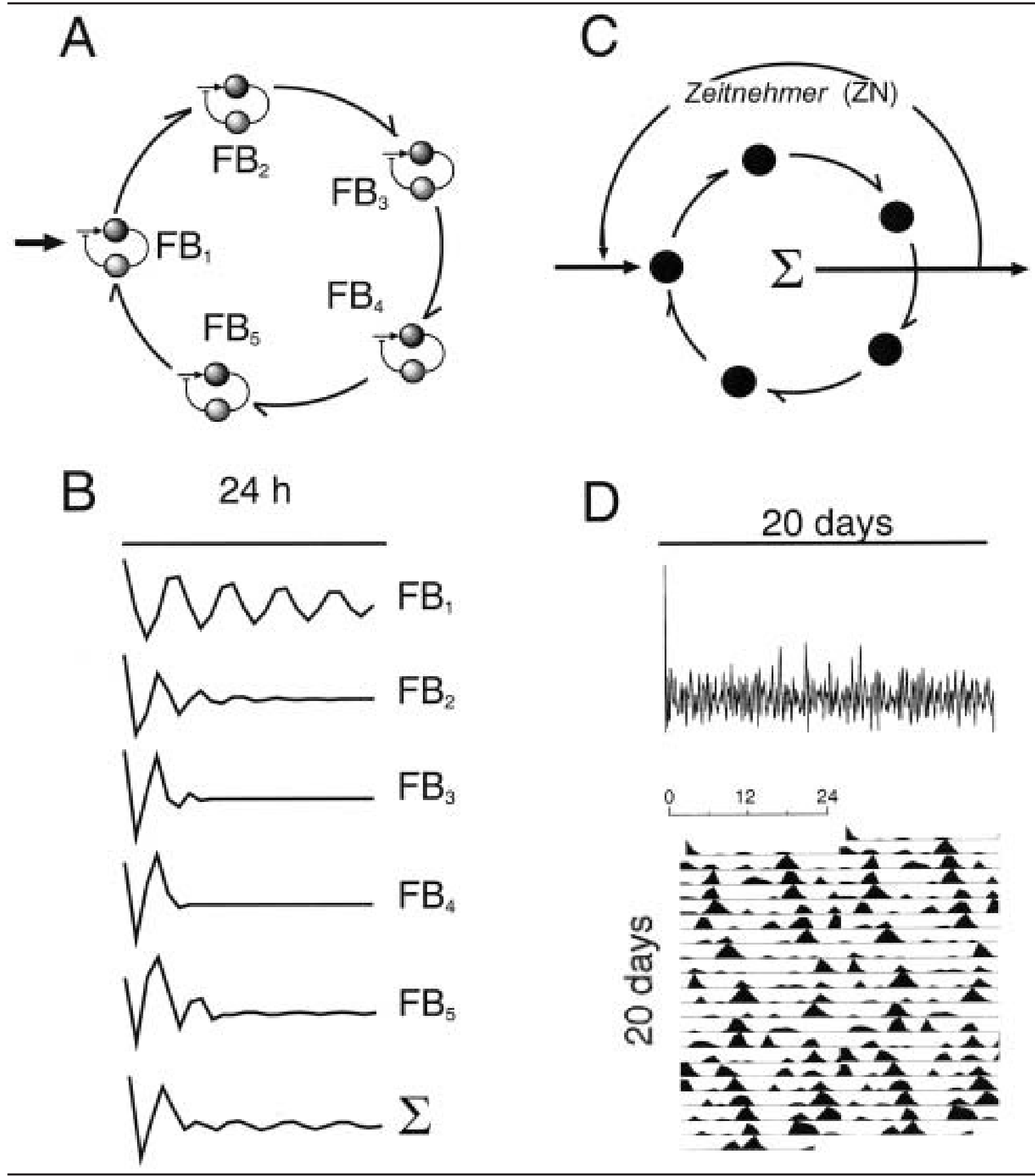

Figure 1. Rhythmicity and synchronization of a model representing a theoretical organism with complex metabolism but without a circadian system. (A) Five negative feedback (FB) loops (for model algorithms) exemplify the numerous FBs in different parts of metabolism. The five FBs are identical except for the turnover of state variable $S_{1}$. (B) Individually and uncoupled, each of the FBs shows an oscillation that dampens more or less rapidly. The top five time series in B represent concentration changes of $S_{2}$ in each of the five FBs. The bottom trace represents the sum of all $S_{2}$ concentrations at a given time $(\Sigma) .(C, D)$ When connected to a circular network, $\Sigma$ is arrhythmic (see linear and double plot representation in D).

or neuronal circuits). Any metabolic network must, therefore, involve numerous negative feedback loops.
The five feedback loops in our model (Fig. 1A) are based on the same set of equations involving the pro- 
Table 1 Variables used to implement the feedback loops, $F B_{1}$ to $F B_{5}$.

\begin{tabular}{ccccccc}
\hline & 1 & 2 & 3 & 4 & 5 & 6 \\
& $\mathrm{r}$ & $\mathrm{p}_{1}$ & $\mathrm{p}_{2}$ & $\mathrm{c}$ & $\mathrm{d}_{1}$ & $\mathrm{~d}_{2}$ \\
\hline$F B_{1}$ & 100 & $Z G+r^{*} e\left(-0.025\left(S_{2,1}+c\right)\right)$ & $S_{1.1}$ & $S_{2,5}$ & $2 / 3 r+0.018 S_{1,1}$ & $Z N+4+0.018 S_{2,1}$ \\
$F B_{2}$ & 200 & $r^{*} e\left(-0.025\left(S_{2,2}+c\right)\right)$ & $S_{1,2}$ & $S_{2,1}$ & $2 / 3 r+0.018 S_{1,2}$ & $4+0.018 S_{2,2}$ \\
$F B_{3}$ & 300 & $r^{*} e\left(-0.025\left(S_{2,3}+c\right)\right)$ & $S_{1,3}$ & $S_{2,2}$ & $2 / 3 r+0.018 S_{1,3}$ & $4+0.018 S_{2,3}$ \\
$F B_{4}$ & 400 & $r^{*} e\left(-0.025\left(S_{2,4}+c\right)\right)$ & $S_{1,4}$ & $2 S_{2,3}$ & $2 / 3 r+0.018 S_{1,4}$ & $4+0.018 S_{2,4}$ \\
$F B_{5}$ & 500 & $r^{*} e\left(-0.025\left(S_{2,5}+c\right)\right)$ & $S_{1,5}$ & $2 S_{2,4}$ & $2 / 3 r+0.018 S_{1,5}$ & $4+0.018 S_{2,5}$ \\
\hline
\end{tabular}

NOTE: $r=$ turnover rate, $p_{1}=$ production rate of state variable $S_{1}, d_{1}=$ degradation rate of state variable $S_{1}, c=$ connectivity between the individual feedbacks, $p_{2}=$ production rate of state variable $S_{2}, d_{2}=$ degradation rate of state variable $S_{2}, \mathrm{ZG}=$ zeitgeber, $\mathrm{ZN}=$ zeitnehmer.

duction and degradation of two state variables $\left(S_{1}\right.$ and $S_{2}$ ) (Roenneberg and Merrow, 1998, 1999). The two state variables could, for example, represent RNA and protein in a TTL or any other two-step negative feedback. The turnover rates of $S_{1}$ (see $r$ in Table 1) are given different values based on the rationale that individual TTLs or metabolic feedback loops are bound to have different qualities. When the five loops are not connected in a network, $F B_{1}$ to $F B_{5}$ show different kinetics of a dampened oscillation with distinct periods in the ultradian range (Fig. 1B). In our model, we have chosen a network of five feedback loops, but similar results can be achieved with three (data not shown). In presuming that each of the feedbacks is directly or indirectly involved in changing a metabolic variable (e.g., $\mathrm{Ca}^{++}$or redox) (Johnson et al., 1995; Merrow and Roenneberg, 2001; Rutter et al., 2001), an integrated oscillation was calculated by simply adding the concentrations of each state variable $S_{2}$ at any given time (Fig. $1 \mathrm{C}$ and $\Sigma$ in Fig. 1B).

When the individual feedback loops are connected in a network, they become self-sustained (i.e., the integrated output does not converge to a steady state as does each of the individual loops when not connected). The system's periodicity, however, is unstable-still in the ultradian range. A long-term recording of the integral of the $S_{2}$ values $(\Sigma)$ shows a chaotic oscillation insofar as the pattern never repeats (tested for 200 "days"; data not shown). The recording resembles the activity patterns of arrhythmic organisms (Fig. 1D). Arrhythmic activity (e.g., Ewer et al., 1990; Kume et al., 1999; Ralph et al., 1990) is generally thought not to be generated by underlying circadian or ultradian oscillators. However, if some readout occurs in bouts, even at irregular intervals, some underlying mechanism must be responsible for these episodes. The "actogram" in Figure 1D supports the notion that oscillators that by themselves are regular but dampened can form a network where they become irregular but self-sustained. In fact, very early on in analyzing the activity patterns of $\mathrm{per}^{0}$ flies, it became clear that the arrhythmicity was much more systematic than could be expected from inspection (Dowse et al., 1987).

\section{Synchronizing Networks to a Cyclic Environment}

Given an appropriate input pathway, each of the simple, unconnected feedback loops is driven by a zeitgeber (as shown for $F B_{1}$ in Fig. 2A). When the feedbacks form a network, however, neither the individual oscillations nor the integrated rhythm is drivable with any stable precision (Figs. 2B, 2C). Their temporal pattern (phase and amplitude) changes every cycle, although concentrations are generally above the daily average during a similar phase of the zeitgeber. Note that this nonsustained system systematically changes phase with zeitgeber strength as do circadian systems (compare Fig. 2B and Fig. 2C). Small but systematic phase angle differences can also be seen when the length of the zeitgeber cycle is varied (data not shown). Phase angle differences due to different zeitgeber strengths or lengths are formally attributed to, and can be predicted from, the phase response curve of an intact system. The results shown here are remarkable in that phase response curves cannot be constructed with the classical protocols (i.e., single perturbations given at different circadian times of a free run in constant conditions).

The results shown in Figure 2 indicate that the complex network is in a different state every time the zeitgeber goes on or off, due to its inherent dynamics, leading to a different response and, thus, resulting in unreliable synchronization. One possibility to counteract unreliable synchronization of the network is to 


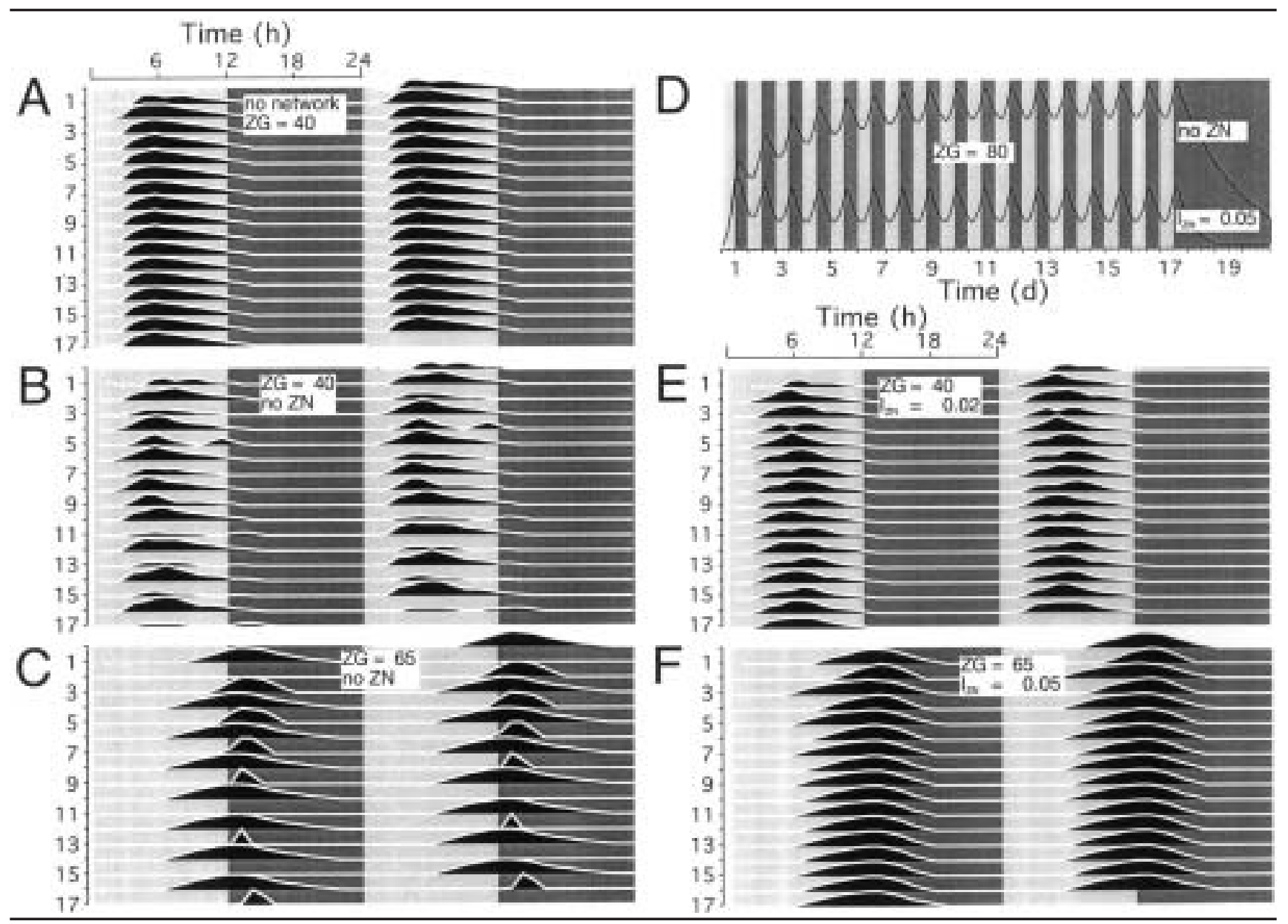

Figure 2. Unreliable synchronization of coupled feedback (FB) loops is overcome with an input FB (zeitnehmer [ZN]). (A) The unconnected FB loops are reliably synchronized by zeitgeber $(Z G)$ cycles (shown for $F B_{1}$; in all plots, "night" is shaded and indicates $Z G=0$ ). $(B$, $C, D)$ When the FBs are connected, however, the network is not reliably synchronized (with different ZG amplitudes as indicated) except at high ZG amplitudes (see also Fig. 3). (E, F) By making the input FB $\left(F B_{1}\right)$ depend on the integral activity of the system (zeitnehmer [ZN] loop), reliable synchronization is achieved.

make the input pathway itself (represented in our model by $\left.F B_{1}\right)$ sensitive to the integrated oscillation $(\Sigma$; Fig. 1C). This additional, global feedback modulates the component of the network that receives the zeitgeber (time giver) and, thus, serves as zeitnehmer (time taker) (Roenneberg et al., 1998). By closing the zeitnehmer loop, the system becomes stably entrained (compare panels $\mathrm{E}$ and $\mathrm{F}$ with $\mathrm{B}$ and $\mathrm{C}$ in Fig. 2). Note, that even with a closed zeitnehmer loop, the system remains arrhythmic in constant conditions (data not shown, but similar to the zeitnehmer-less network shown in Fig. 1D)—clearly not an accurate representation of a circadian system. The stabilizing effect of a zeitnehmer is also seen when all feedback loops are given the same ( $r=100$; data not shown), rather than different, turnover rates (see Table 1).
Stable synchronization depends on zeitgeber strength. Systems obviously do not synchronize when a zeitgeber is too weak, but they may also lose synchronization when it is too strong (Gonze and Goldbeter, 2001). Does the zeitnehmer loop stabilize the network so that synchronization occurs over a wider range of zeitgeber strengths, or does it simply reduce the impact of the zeitgeber? We studied the effects of different zeitgeber and zeitnehmer strengths systematically. At high zeitgeber amplitudes, even the zeitnehmer-less network can be synchronized; however, concentrations increase steadily over time until they oscillate around a high mean level (Fig. 2D). This trend is absent when the zeitnehmer loop is closed. Due to the inherent kinetics of the network, synchronization breaks down at intermediary zeitgeber 

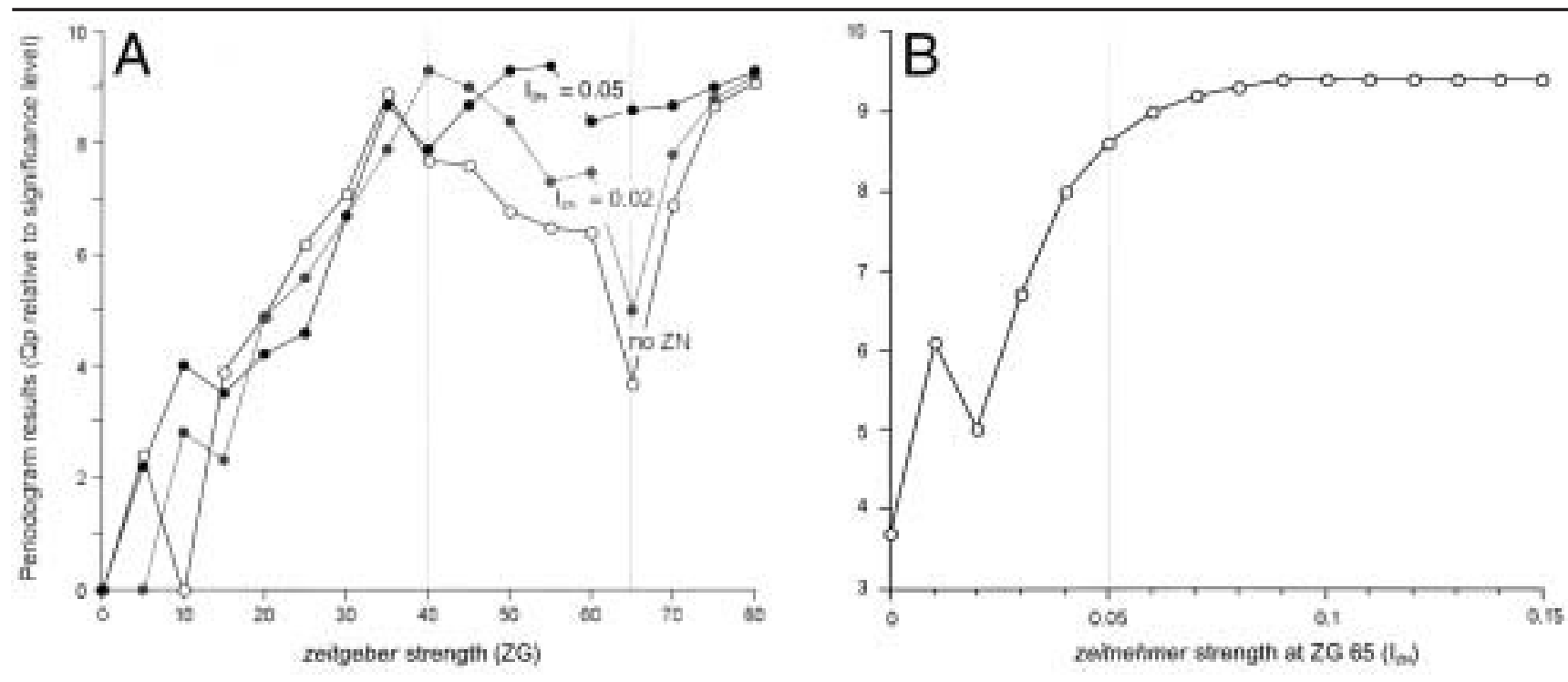

Figure 3. Synchronization, zeitgeber, and zeitnehmer (ZN) strength. (A) Due to the kinetics of the ZN-less network, synchronization is unreliable. Between zeitgeber strengths 40 and 75, the system starts to skip cycles (open symbols). (B) This breakdown is stabilized when the ZN loop is closed, depending on its impact on the input feedback, $F B_{1}$ (see different $Z N$ strength, $I_{Z N}$, in panel A and panel B for a fixed zeitgeber strength of 65). The ordinates show quality of entrainment, judged by periodogram analysis (Qp value at $T=24)($ Sokolove and Bushell, 1978) and plotted relative to the chi-square significance level (1 representing $p \leq 0.01$ and 0 when the periodogram shows no peak around $T=24)$.

strength (around $Z G=65 ;$ Fig. 3A). This breakdown can be prevented by increasing the impact of the zeitnehmer loop (Fig. 3B).

\section{From Synchronization to Entrainment}

Evolution is thought to proceed in small steps rather than in big jumps (Dawkins, 1997). We propose that the ancestral components from which a circadian clock evolved consisted of mechanisms that enabled complex cellular networks to be driven by environmental cycles. From these, only small changes may have been necessary to turn a drivable network that is arrhythmic in constant conditions (Fig. 1D) into an entrainable, self-sustained circadian system (Fig. 4). In our model, we exemplified this small "evolutionary" step by simply strengthening the connectivity between $F B_{5}$ and $F B_{1}$ (changing it from 1 to 6). Under these conditions, the network becomes self-sustained in the circadian range (with an average $\tau$ of $17.3 \mathrm{~h}$; Figs. 4A, 4B). With stronger connectivity in parts of the network, the system is even less apt to be driven without the compensating effect of a zeitnehmer loop (Fig. 4C). When the zeitnehmer loop is closed, the period of the free-running integrated rhythm depends on zeitnehmer strength-the stronger its impact, the shorter the period (data not shown). This leads to the fact that the entrained network adopts different phase angles with different zeitnehmer strengths and even shows transients typical for circadian systems (Figs. 4D, 4E).

In the model used here, changes in entrainment phase can be achieved simply by varying the connectivity between the individual feedback loops of a complex network or by altering the strength of the zeitnehmer loop (Figs. 4D, 4E). The circadian systems of both day- and night-active organisms (e.g., mouse and man) are apparently built by the same components, and even within the same organism, day and night activity can change depending on conditions. Day-active migratory birds, for example, which travel long distances at night, change their activity phase at the appropriate times of the year (Gwinner, 1996), and humans, which are "larks" both as a child and as an adult, tend to be "owls" during adolescence (Carskadon et al., 1998). It is likely that such developmental or history-dependent changes in entrainment phase are due to physiological/metabolic changes rather than to changes in the genetic clock components. In our model, these changes are implemented by changing the impact of networked components on 

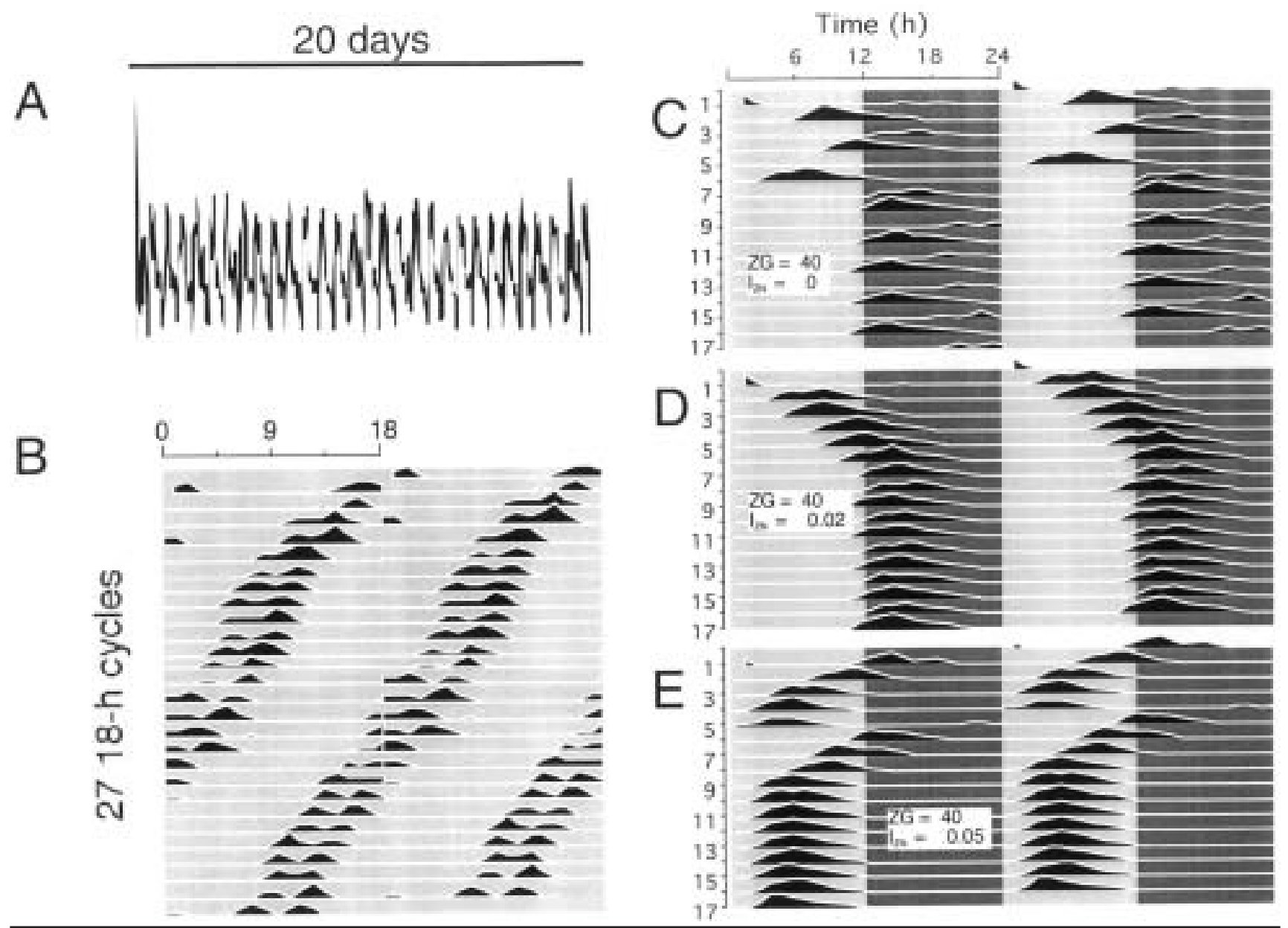

Figure 4. The theoretical organism represented in our model "evolves" from an arrhythmic to a circadian system by strengthening the connectivity between two feedback loops. In this case, the connectivity between the feedback loops with the highest $\left(F B_{5}\right)$ and lowest $\left(F B_{1}\right)$ turnover rate was changed from 1 to 6 . (A, B) Under these conditions, the system free-runs with a period of approximately $17 \mathrm{~h}(\mathrm{~A}$ is a linear plot, and B is a double plot with $18 \mathrm{~h}$ modulo $\tau$ ). (C) Due to this stronger connectivity, the system becomes even less drivable by a zeitgeber (ZG) cycle (compare with panels B and C in Fig. 2). (D, E) However, when the zeitnehmer (ZN) loop is closed, the system is not only reliably synchronized by the zeitgeber but shows additional qualities of circadian entrainment, such as transients (D, E). The phase of stable entrainment differs depending on how much the ZN loop affects the input feedback, $F B_{1}$.

each other, resulting in different production and degradation rates. The model suggests that the circadian qualities currently attributed to a single TTL could also be implemented by multiple feedback loops with noncircadian periods connected in a network. The idea that a circa-24-h rhythmicity may be a product of coupled ultradian oscillators was put forward approximately 25 years ago (Dowse and Ringo, 1987) but has been overwhelmed by the apparent power in explaining circadian qualities by the interactions of a handful of clock genes in a TTL (Darlington et al., 1998; Reppert and Weaver, 2001). Now that different clock mutants are being submitted to more rigorous physiological analysis, we are gathering more detailed information about exactly which circadian qualities are defective in a given clock mutant or in combinations of clock mutants, and multiple oscillator models may be revisited.

\section{Experimental Predictions}

Models such as this one are only useful if they produce experimental ideas. With weaker direct coupling in parts of the circuit, the model simulates an arrhythmic organism (Fig. 2), whereas increasing coupling strength leads to an output resembling that of a circadian organism (Fig. 4). The main difference between the two model versions lies in the fact that the latter consolidates a circadian rhythmicity in the absence of a zeitgeber whereas the former does not. Under entrained conditions, the two models behave similarly, except for the typical circadian transients 
present in both the self-sustained models. Circadian research has traditionally based many of its experiments on the self-sustainment in constant conditions, whereas the presented model makes a strong case for experiments using systematic entrainment protocols to probe for the mechanisms underlying circadian behavior. The necessity of employing entrainment conditions in investigating circadian systems was often stressed by Jürgen Aschoff.

If circadian systems were built on mechanisms that ensure complex metabolism to be drivable by zeitgebers, then the components of this mechanism can be identified in organisms without self-sustained circadian rhythmicity. Such organisms include those that (1) did not evolve a circadian system, (2) express self-sustained rhythmicity only in as-yet undiscovered outputs or only under specific constant conditions that have not been found, or (3) have been "made" arrhythmic by mutation. The first two classes are difficult to separate because the lack of selfsustained circadian rhythmicity may only be due to the fact that the right conditions or the right output variables have not yet been discovered. Cyanobacteria and Caenorhabditis elegans are good examples of organisms that were historically regarded as "clockless" but were subsequently found to be circadian (Johnson et al., 1996; Kippert et al., 2002; Saigusa et al., 2002). Our model results predict that circadian components should exist in all three classes of arrhythmic organisms.

The model predicts that organisms that are arrhythmic in constant conditions show circadian properties under entrained conditions. Systematic phase relationships should be measurable in any of the clockless organisms for changing both zeitgeber lengths and strengths, and arrhythmicity should be conditional in many of them. These predictions can easily be tested in organisms of all three classes mentioned above, and some confirming results already exist. Systematic phase angles in zeitgeber cycles of different amplitudes and lengths have already been shown for clock mutants in Neurospora (Merrow et al., 1999), and release experiments show remaining circadian qualities in Arabidopsis clock mutants (McWatters et al., 2000). Neurospora clock mutants that are initially arrhythmic develop a self-sustained rhythm when grown on long race tubes (Aronson, Johnson, and Dunlap, 1994; Loros and Feldman, 1986), and mouse clock mutants are arrhythmic in constant darkness but not in constant light (Steinlechner et al., 2002). Inter- estingly, the model shown in Figure 2 can also be made self-sustained when constant light is simulated (data not shown).

In addition, mutagenesis of putative clockless organisms or further mutagenesis of clock mutants should reveal additional components beyond the known TTLs. A reporter gene could be used to monitor key metabolic outputs, for example, in temperature cycles. Prior to mutagenesis, expression patterns under zeitgeber conditions should be similar to those shown in Figures 2E and 2F (reliable synchronization). Following mutagenesis, one would screen for mutants with a phenotype similar to those shown in Figures 2B and 2C (unreliable synchronization). Once genes have been identified that enable complex metabolic networks to be synchronized by zeitgebers, their homologues can be deleted in organisms with an intact circadian system to investigate their role in the circadian machinery. Because several of these components may be important for both the (extended) clock system and for viability, strategies have to be applied to prevent lethal mutations (e.g., overexpression). With growing possibilities of coping experimentally with complex systems, we will be able to incorporate components that contribute to the circadian phenotype in a less legotype fashion than before.

\section{ACKNOWLEDGMENTS}

Our work is supported by the Eppendorf Company, Hamburg, the Deutsche Forschungsgemeinschaft, the Dr.-Meyer-Struckmann-Stiftung, and the FriedrichBaur-Stiftung.

\section{REFERENCES}

Achermann P and Kunz H (1999) Modeling circadian rhythm generation in the suprachiasmatic nucleus with locally coupled self-sustained oscillators: Phase shifts and phase response curves. J Biol Rhythms 14:460-468.

Alabadi D, Oyama T, Yanovsky MJ, Harmon FG, Mas P, and Kay SA (2001) Reciprocal regulation between TOC1 and LHY/CCA1 within the Arabidopsis circadian clock. Science. 293:880-883.

Albrecht U, Zheng B, Larkin D, Sun ZS, and Lee CC (2001) mPer 1 and mPer 2 are essential for normal resetting of the circadian clock. J Biol Rhythms 16:100-104.

Aronson BD, Johnson KA, and Dunlap JC (1994) The circadian clock locus frequency: A single ORF defines period length and temperature compensation. Proc Natl Acad Sci U S A 91:7683-7687. 
Aronson BD, Johnson KA, Loros JJ, and Dunlap JC (1994) Negative feedback defining a circadian clock: Autoregulation of the clock gene frequency. Science 263:1578-1584.

Bargiello TA, Jackson FR, and Young MW (1984) Restoration of circadian behavioural rhythms by gene transfer in Drosophila. Nature 312:752-754.

Blau J (2001) The Drosophila circadian clock: What we know and what we don't know. Semin Cell Dev Biol 12:287-294.

Carskadon MA, Wolfson AR, Acebo C, Tzischinsky O, and Seifer R (1998) Adolescent sleep patterns, circadian timing, and sleepiness at a transition to early school days. Sleep 21:871-881.

Crosthwaite SK, Dunlap JC, and Loros JJ (1997) Neurospora $w c-1$ and $w c-2$ : Transcription, photoresponses, and the origin of circadian rhythmicity. Science 276:763-769.

Daan S (1981) Adaptive daily strategies in behavior. In Biological Rhythms, J Aschoff, ed, pp 275-298, Plenum Press, New York.

Daan S, Albrecht U, van der Horst GTJ, Illnerova $H$, Roenneberg T, Schwartz WJ, and Wehr TA (2001) Assembling a clock for all seasons: Are M and E oscillators in the genes? J Biol Rhythms 16:105-116.

Darlington TK, Wager-Smith K, Ceriani MF, Staknis D, Gekakis N, Steeves TDL, Weitz CJ, Takahashi JS, and Kay SA (1998) Closing the circadian loop: CLOCK-induced transcription of its own inhibitors per and tim. Science 280:1599-1603.

Dawkins R (1997) Climbing Mount Improbable, WW Norton, London.

Dowse HB, Hall JC, and Ringo JM (1987) Circadian and ultradian rhythms in period mutants of Drosophila melanogaster. Behav Gen 17:19-35.

Dowse HB and Ringo JM (1987) Further evidence that the circadian clock in Drosophila is a population of coupled ultradian oscillators. J Biol Rhythms 2:65-76.

Ewer J, Hamblen-Coyle M, Rosbash M, and Hall JC (1990) Requirement for period gene expression in the adult and not during development for locomotor activity rhythms of imaginal Drosophila melanogaster. J Neurogenetics 7:31-73.

Gekakis N, Staknis D, Nguyen HB, Davis FC, Wilsbacher LD, King DP, Takahashi JS, and Weitz CJ (1998) Role of the CLOCK protein in the mammalian circadian mechanism. Science 280:1564-1569.

Gonze D and Goldbeter A (2001) A model for a network of phosphorylation-dephosphorylation cycles displaying the dynamics of dominoes and clocks. J Theor Biol 210:167-186.

Gwinner E (1996) Circadian and circannual programmes in avian migration. J Exp Biol 199:39-48.

Hardin PE, Hall JC, and Rosbash M (1990) Feedback of the Drosophila period gene product on circadian cycling of its messenger RNA levels. Nature 343:536-540.

Herzog ED and Tossini G (2001) The mammalian circadian clock shop. Semin Cell Dev Biol 12:295-304.

Iwasaki $\mathrm{H}$ and Kondo T (2000) The current state and problems of circadian clock studies in cyanobacteria. Plant Cell Physiol 41:1013-1020.

Johnson CH, Golden SS, Ishiura M, and Kondo T (1996) Circadian clocks in prokaryotes. Mol Microbiol 21:5-11.
Johnson CH, Knight MR, Kondo T, Masson P, Sedbrook J, Haley A, and Trewavas A (1995) Circadian oscillations of cytosolic and chloroplastic free calcium in plants. Science 269:1863-1865.

Kippert F, Saunders DS, and Blaxter ML (2002) Caenorhabditis elegans has a circadian clock. Curr Biol 12:R47-R49.

Kume K, Zylka MJ, Sriram S, Shearman LP, Weaver DR, Jin X, Maywood ES, Hastings MH, and Reppert SM (1999) mCRY1 and mCRY2 are essential components of the negative limb of the circadian clock feedback loop. Cell 98:193-205.

Lakin-Thomas PL and Brody S (2000) Circadian rhythms in Neurospora crassa: Lipid deficiencies restore robust rhythmicity to null frequency and white-collar mutants. Proc Natl Acad Sci U S A 97:256-261.

Leloup JC and Goldbeter A (1998) A model for circadian rhythms in Drosophila incorporating the formation of a complex between the PER and TIM proteins. J Biol Rhythms 13:70-87.

Loros JJ and Dunlap JC (2001) Genetic and molecular analysis of circadian rhythms in Neurospora. Ann Rev Physiol 63:757-794.

Loros JJ and Feldman JF (1986) Loss of temperature compensation of circadian period length in the frq-9 mutant of Neurospora crassa. J Biol Rhythms 1:187-198.

McWatters HG, Bastow RM, Hall A, and Millar AJ (2000) The ELF3 zeitnehmer regulates light signalling to the circadian clock. Nature 408:716-720.

Merrow M, Brunner M, and Roenneberg T (1999) Assignment of circadian function for the Neurospora clock gene frequency. Nature 399:584-586.

Merrow M and Roenneberg T (2001) Circadian clocks: Running on redox. Cell 106:141-143.

Merrow M, Roenneberg T, Macino G, and Franchi L (2001) A fungus among us: The Neurospora crassa circadian system. Semin Cell Dev Biol 12:279-285.

Mori T and Johnson CH (2001) Circadian programming in cyanobacteria. Semin Cell Dev Biol 12:271-278.

Oda GA, Menaker M, and Friesen WO (2000) Modeling the dual pacemaker system of the tau mutant hamster. J Biol Rhythms 15:246-264.

Page TL, Mans C, and Griffeth G (2001) History dependence of circadian pacemaker period in the cockroach. J Insect Physiol 47:1085-1093.

Petri B and Stengl M (2001) Phase response curves of a molecular model oscillator: Implications for mutual coupling of paired oscillators. J Biol Rhythms 16:125-141.

Ralph MR, Foster RG, Davis FC, and Menaker M (1990) Transplanted suprachiasmatic nucleus determines circadian period. Science 247:975-978.

Reppert SM and Weaver DR (2001) Molecular analysis of mammalian circadian rhythms. Ann Rev Physiol 63:647676.

Roenneberg T and Merrow M (1998) Molecular circadian oscillators-An alternative hypothesis. J Biol Rhythms 13:167-179.

Roenneberg T and Merrow M (1999) Circadian clocks and metabolism. J Biol Rhythms 14:449-459. 
Roenneberg T and Merrow M (2001) Circadian systems: Different levels of complexity. Phil Trans Roy Soc Lond 356:1687-1696.

Roenneberg T, Merrow M, and Eisensamer B (1998) Cellular mechanisms of circadian systems. Zoology 100:273-286.

Roenneberg T and Taylor W (2000) Automated recordings of bioluminescence with special reference to the analysis of circadian rhythms. Methods Enzymol 305:104-119.

Rutter J, Reick M, Wu LC, and McKnight SL (2001) Regulation of Clock and NPAS2 DNA binding by the redox state of NAD cofactors. Science 293:510-514.

Saigusa T, Ishizaki S, Watabiki S, Ishii N, Tanakadate A, Tamai Y, and Hasegawa K (2002) Circadian behavioural rhythm in Caenorhabditis elegans. Curr Biol 12:R46-R47.

Sokolove PG and Bushell WN (1978) The chi square periodogram: Its utility for analysis of circadian rhythms. J Theor Biol 72:131-160.

Stanewsky R, Kaneko M, Emery P, Beretta B, Wagner-Smith K, Kay SA, Rosbash M, and Hall JC (1998) The cry ${ }^{b}$ mutation identifies cryptochrome as a circadian photoreceptor in Drosophila. Cell 95:681-692.
Steinlechner S, Jacobmeier B, Scherbarth F, Dernbach H, Kruse F, and Albrecht U (2002) Robust circadian rhythmicity of Per1 and Per2 mutant mice in constant light and dynamics of Per1 and Per2 gene expression under long and short photoperiods. J Biol Rhythms 17:202-209.

Taylor W, Krasnow T, Dunlap JC, Broda H, and Hastings JW (1982) Critical pulses of anisomycin drive the circadian oscillator in Gonyaulax towards its singularity. J Comp Physiol B 148:11-25.

Williams JA and Sehgal A (2001) Molecular components of the circadian system in Drosophila. Ann Rev Physiol 63:729-755.

Winfree AT (1970) An integrated view of the resetting of a circadian clock. J Theor Biol 28:327-374.

Zheng B, Albrecht U, Kaasik K, Sage M, Lu W, Vaishnav S, Li Q, Sun ZS, Eichele G, Bradley A, and Lee CC (2001) Nonredundant roles of the mPer 1 and $m$ Per 2 genes in the mammalian circadian clock. Cell 105:683-694. 